\title{
ORCHESTRATING TOURISM ACTORS' NETWORK VIA THE "N-1 N+1 TOUCHPOINTS" ALGORITHM: A B2B CHATBOT TO IMPROVE CUSTOMER'S JOURNEYS
}

\author{
Randolf Ramseyer, Davide Calvaresi, Benjamin Nanchen, Roland Schegg, Michael Schumacher \\ and Emmanuel Fragnière \\ University of Applied Sciences Western Switzerland (HES-SO Valais-Wallis) \\ Rue de la Plaine 2, 3960 Sierre, Switzerland
}

\begin{abstract}
This paper elaborates on a novel concept to orchestrate tourism networks. In particular, each actor optimizes his service (touchpoint $\mathrm{n}$ ) while seamlessly transferring the customer information from the previous (touchpoint $\mathrm{n}-1$ ) to the following service (touchpoint $\mathrm{n}+1$ ). To implement such a theory, we leveraged on chatbot technology, which interfacing directly with the user eased the transition from the point of interaction (touchpoint) $n-1$ to $n+1$. Moreover, the chatbot entails the connection between the nodes of the customer's journey, enabling user profiling, personalization, and knowledge transfer. The deployment of a chatbot implementing the "n-1 n+1 touchpoints" model would significantly benefit actors operating in a fragmented touristic economy (i.e., Switzerland). Hence, we tested the first prototype in the heart of the Canton Valais, where, in collaboration with students in tourism, we based the scenario on counterfactual thinking. In particular, we identified all the possible situations a grandmother and her grandson might face arriving at the Sierre train station to spend a day in Crans-Montana. In turn, using reenactment theatre techniques, tourism professionals played the worst-case scenario (without chatbot) and the best case (with the chatbot) to elicit the different clients' perceptions on those diametral situations. Such a feasibility study paved the way to a more holistic view, employing artificial intelligence techniques to enhance the chatbot and smoothen the "n-1 n+1 touchpoints" dynamics.
\end{abstract}

\section{KEYWORDS}

Counterfactual Analysis, Customer Journey, Chatbot, Fragmented Touristic Network, Touchpoint, Artificial Intelligence

\section{INTRODUCTION}

Technologies enabling personalized interactions can operate the virtual management of the information among the touchpoints, duly complying with privacy-preserving mechanisms. Indeed, the sole information envisioned to be transferred in the chain $(n-1)-(n)-(n+1)$ are functional to a given service and must comply with the visibility constraints (see Figure 1).

Equipping the "n-1 n+1 concept" with chatbot technologies and Artificial Intelligence (AI) techniques can enable a "self-orchestration" of the entire customer journey, enabling a seamless harmonization of the tourist actors and strengthening anti-intrusion mechanisms. For example, a carrier (n-1) can inform a sports shop that a certain number of customers are approaching to rent skis. The sports shop can be able to inform the respective hotels $(n+1)$ that the rented ski equipment will be delivered to their doorstep, allowing the customers to freely go shopping in the resort after they visited the shop. This "digital takeover" model represents a great potential for automated assistance in the planning phase, during a stay, and afterward. Coupling each tourist with a personalized virtual entity can ensure smooth and secure (i.e., via seamless authentification) interactions and autonomous interconnections among the possible nodes of the customers' itineraries, widening the opportunities for innovation.

To this end, this paper addresses how and why chatbots can bring a real advantage to the optimization of the customer journey in a fragmented touristic economy (i.e., like Switzerland), as well as how to integrate the chatbot technology pragmatically and reliably, directly under the line of visibility (separation between the back-office and the front-office in a service blueprint, see Shostack, 1984) so as a B2B tool. 
The context of this investigation takes into account elements of short touristic network (SNA). For example, see (Racherla and $\mathrm{Hu}, 2010$ ), references to the scientific literature to chatbots (Calvaresi et al., 2021) and counterfactual thinking (Gaglio 2004). Our service design methodology (Fragnière et al., 2012) starts from hypothetical scenarios of a persona's practical problems possibly encountered in his/her typical customer journey (i.e., on the way to a resort). Then, this customer journey involving 'points of failure' is integrated into a 'service blueprint' (shostack, 1984) to link it to the different points of contact (or points of interaction) with the different tourism actors (or service providers). Finally, a real simulation is conducted, and pragmatic solutions are proposed to establish a practical link with the back office of this digital takeover system (i.e., the chatbot app implementing the "n-1 n+1" touchpoints algorithm). Finally, the paper concludes by presenting the last stage of this ongoing research (i.e., integrating AI in the chatbot platform) which will be tested with the tourist office of Sierre.

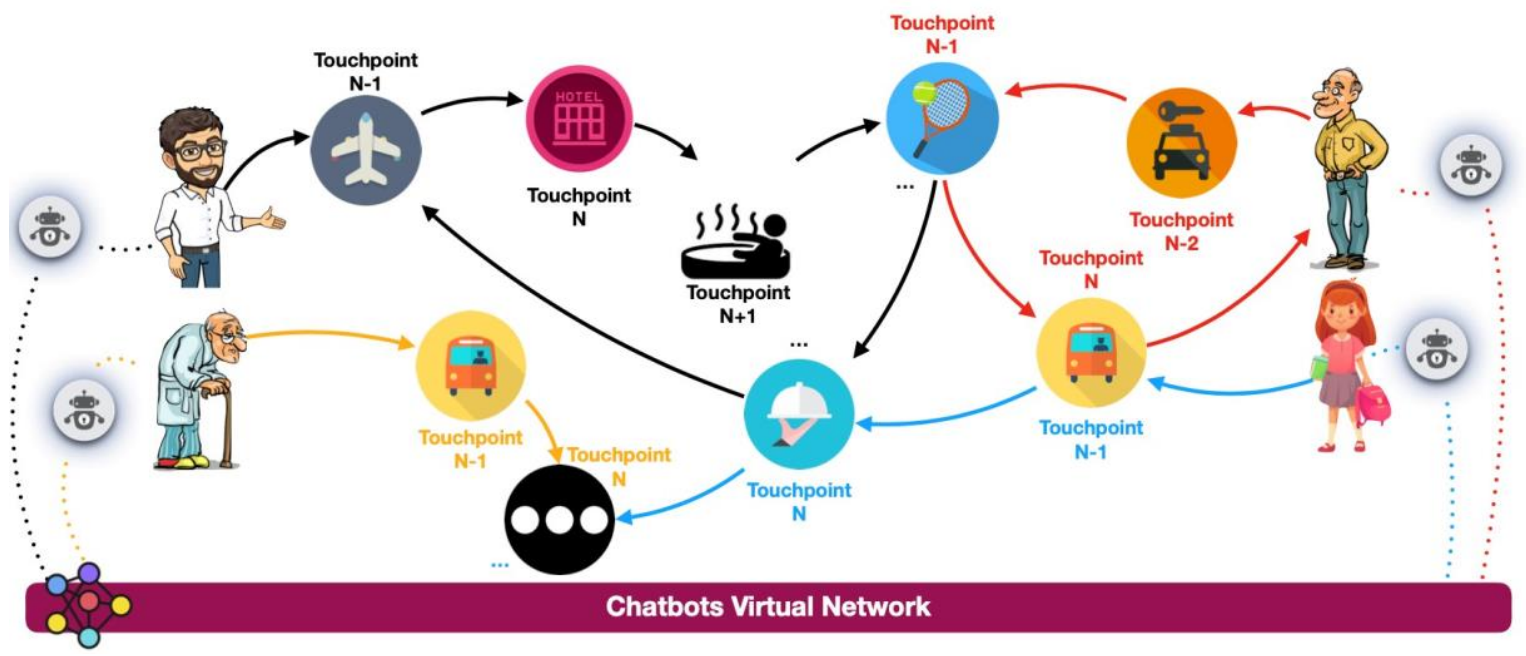

Figure 1. N-1 N+1 Concept Schematization

\section{IMPLEMENTING THE N-1 N+1 TOUCHPOINT IN A B2B CHATBOT}

The business as usual scenario and starting scenario follows. A grandmother and her grandson plan to spend a day in Crans-Montana. They arrive in Sierre by train at $10.34 \mathrm{am}$. Yet, they do not know the way to Crans-Montana. However, they certainly know that they have to be back in Sierre by 16:24 to catch that very train (Supersaver ticket). At the end of the day, they had a good time together. Nevertheless, everyone complains about having waited and walked more than they wanted to. In terms of $n-1$ and $n+1$, the business as usual scenario becomes:

n-1: They took the bus from Sierre to Montana (they saw that there is a funicular to get off afterward).

n: They had lunch in a hamburger restaurant -- the grandson loves hamburgers. As a matter of fact, they saw the Burger Lounge only after walking around Crans for 30 minutes. By then, no table was available. Thus, they had to go back to the restaurant an hour later.

$\mathbf{n + 1}$ : Having seen a funicular nearby, they decided to take it for the way down. They arrived at 14:50 at the Montana station, took the funicular at 15:15, and arrived in Sierre at 15:35. Once they walked to the railway station of Sierre, they had to wait until 16.24 to benefit from the supersaver ticket solely valid for that train.

The primary goal is to stage this $n-1 n+1$ concept in a real setting. Thus, based on this scenario, our BSc in Tourism students took into account all existing constraints and opportunities (train times, grandmother's reduced mobility, grandson's desires, etc.). In turn, they identified (actual) n-1 and n+1 possibilities (good and bad) based on counterfactual thinking patterns. Based on these $n-1 n+1$ possibilities, we have chosen an optimized scenario to be programmed in the chatbot. As a next step, we plan to incorporate AI algorithms to generate several $n-1 n+1$ paths for the clients (tailored to the customer profile) automatically. 
The optimized scenario obtained by using the chatbot under the line of visibility (back-office) and the n-1 and $\mathrm{n}+1 \mathrm{~B} 2 \mathrm{~B}$ logic follows:

n-1: When they arrive in Sierre, a CMC virtual agent (chatbot operating on behalf of the funicular and buses firm) asks them where they intend to go and, based on their desire, it advises them to take the funicular instead of the bus by following the red line at the exit of the train station (there is a red line painted on the road connecting the station to the funicular). The agent also asks them what they want to do in Crans-Montana and if any suggestion is needed. The grandmother and child welcome such a suggestion, letting the chatbot to contacts the Burger Lounge agent and successfully book a table for two for the time of their arrival. Moreover, having such information, they adjusted the walk accordingly.

n: They arrive with the funicular at Montana Station at 11.00. The funicular driver gives them a map and instructs them on possible shopping path(s) according to their available time. The grandson wants to buy a Crans-Montana T-shirt and cap, so the funicular driver directs them to the Maison du Tourisme in Crans (on the way to Burger Lounge). As planned, at 12.00, they consume the beloved burger. The Burger Lounge's chatbot (aware of the booking) gets in touch again with the tourists, asking if they would have liked other suggestions (compliant with their existing schedule - by then just being able to catch the 16.24 train). Once again, grandmother and grandson welcome the chatbot suggestions, and among them, they opt for booking an 18-hole minigolf court.

$\mathbf{n + 1}$ : A proactive behavior of the tourists' chatbot is triggered by an AI algorithm that crosses their position, the time being, and their schedule. It turns out that the two stay longer than expected at the minigolf bar. At this point, reaching the Funicular Station at a walking-pace as planned is no longer a viable option. Therefore, the chatbot proposes to arrange a small shuttle to reach the Funicular Station. The two agree, and the plan gets promptly put in place.

If they had missed the 15.50 funicular, they would have had to buy a new full price ticket. Yet, thanks to the chatbot's proactive support, they arrive in Sierre on time to catch the train and conclude a great day!

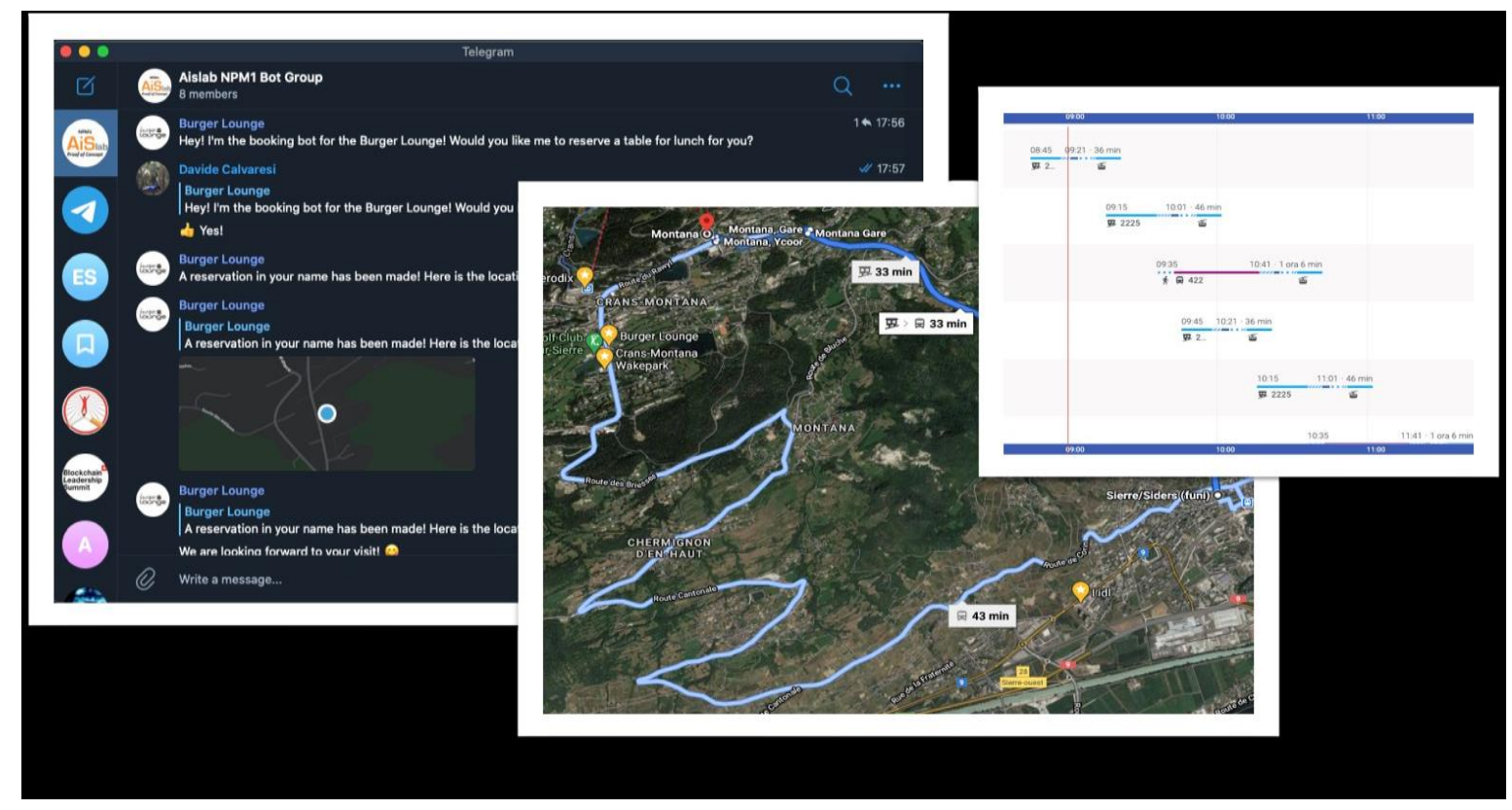

Figure 2. Extract of the chatbot application based on " $n-1 n+1$ touchpoints" and a map of Sierre/Crans-Montana

Figure 2 shows an extract of the chatbot developed at the HES-SO Valais-Wallis, which implements the optimized $n-1 n+1$ scenario described above. It is possible to notice that the information exchange between the various players $(n-1, n$ and $n+1)$ is straightforward and sufficient to satisfy the customer and ensure well-coordinated journeys. Figure 2 also shows on the right a Google map of the two main transport means connecting Sierre to Crans-Montana (i.e., by bus or by funicular, the latter being the fastest and providing a great panoramic experience). Unfortunately, people visiting the region for the first time are often unaware it. 


\section{REAL LIFE STAGING TO TEST THE B2B CHATBOT FEASABILITY}

Once the scenario was programmed on the chatbot, we worked with the Tourism Office of Sierre. Several people played different roles (i.e., grandmother, grandson, funicular driver in Crans-Montana, restaurateur, and owner of the minigolf courts). Along with the play, the human actors interacted with the chatbot.

Given the pandemic circumstances and the need to minimize the contacts, we distributed printed slides representing the possible states of the chatbots and associated illustrated scenarios ${ }^{1}$ (realized by a local artist), see Figure 3. To fully integrate this counterfactual notion, we played, based on a Service Design re-enactment approach (Fragnière et al., 2012). The scene begins at the Sierre railway station according to the schedule established by the scenario as usual on December 9, 2020. The screen is presented as of December 9,2025 , in order to project ourselves into the future. The idea of this first experiment is to stage this B2B chatbot in a real-life experience. Indeed, the dazzling developments of digitalization are not necessarily integrated into the world of tourism and also within society (Durugy, 2020). The actors worked as if they were discovering a play for the first time. They promptly played their role as professionals and were able to compensate for the script's adaptation to reality. Once the role-playing was over, we proceeded with a C-sketching exercise (Shah et al., 2001) as shown in Figure 3, in the middle, to identify all the weaknesses, strengths, and potentials of using a B2B chatbot operating on the "n-1 n+1 touchpoints" principle. We are currently analyzing and exploiting this data to offer the Sierre Tourisme Office and the associated tourist network the first version of a chatbot operating just by exchanging information between $n-1, n$ and $n+1$ touchpoints. When the architecture will be in place and the system operational, the AI algorithms will be integrated to provide the $n-1, n$ and $n+1$ actors with chatbots exposing proactive and autonomous behaviors (i.e., driven by proximity point-of-interest, time, and triggered by previous or following touchpoints).

Tourism professionals have little competence in the digital experience. The advantage of a B2B chatbot rather than a $\mathrm{C} 2 \mathrm{C}$ or $\mathrm{B} 2 \mathrm{C}$ chatbot is that the link between $\mathrm{n}-1$ and $\mathrm{n}+1$ is directly optimized by the virtual agent embodying tourism players, while they can concentrate on the offered services. Second, B2B chatbots do not explicitly require an optimized interface. The envisioned volume of information exchanged is minimal. For example, the funicular agent who sends a shuttle to the minigolf courts to pick up the grandmother and her grandson just needs the number of customers and a position. Once this feasibility has been demonstrated and the AI algorithms integrated, automatic learning mechanisms will be studied to enhance the $n-1$ and $n+1$ model with increasingly tailored and predictive capabilities to boost and possibly anticipate the tourists' needs.

${ }^{1}$ Slides' link: https://drive.switch.ch/index.php/s/OiowKyltAoaSFgt 

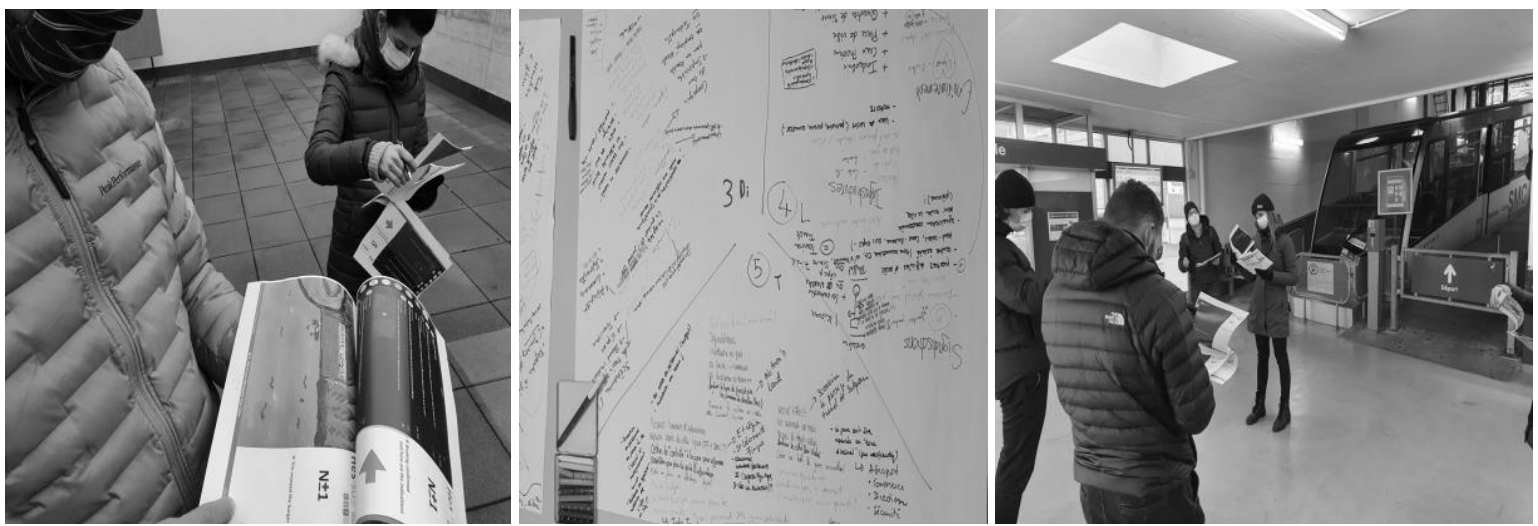

Figure 3. Re-enactement (staging) of the chosen $\mathrm{N}-1 \mathrm{~N}+1$ scenario and C-sketching for feasibility testing purposes

\section{CONCLUSION}

This paper presented the use of chatbot technologies to enable self-orchestration in fragmented tourism economies. In particular, we have integrated into a chatbot for tourism professionals (B2B) a concept we have created called "n-1 n+1 touchpoints" algorithm. The underlying idea is that an actor can engage a customer directly via the chatbot, connecting upstream and downstream service providers seamlessly, enhancing the user experience. The logic of self-orchestration is interesting in scenarios like Switzerland, where the tourism sector is typically very fragmented. Indeed, our study is based on a real-life scenario experienced by most tourists who come for the first time by train to Crans-Montana and experience a very disunite journey. Elaborating such a case study, we have identified a series of $n-1$ and $n+1$ possibilities that enabled us to improve the service's overall quality significantly. Finally, we have designed and integrated an optimized n-1 n+1 scenario into a chatbot prototype deployed for a contextual feasibility study and identified the future steps.

\section{REFERENCES}

Calvaresi, D., Ibrahim, A., Calbimonte, J. P., Schegg, R., Fragniere, E., \& Schumacher, M. (2021). The Evolution of Chatbots in Tourism: A Systematic Literature Review. Information and Communication Technologies in Tourism 2021, pp. 3-16.

Durugy, E., Darouichi, O., \& Fragniere, E., 2020. Staging Blockchain Technology In The Customer's Journey Through Service Design: A Bike-sharing Case Study To Improve Quality And Trust Perception. In Proceedings of the 2nd International Conference on Industrial Control Network And System Engineering Research, pp. 70-76.

Gaglio, C. M., 2004. The role of mental simulations and counterfactual thinking in the opportunity identification process. Entrepreneurship Theory and Practice, Vol. 28, No. 6, pp. 533-552.

Fragnière, E., Nanchen, B., \& Sitten, M., 2012. Performing service design experiments using ethnomethodology and theatre-based reenactment: a Swiss ski resort case study. Service Science, Vol. 4, No. 2, pp. 89-100.

Racherla, P., \& Hu, C., 2010. A social network perspective of tourism research collaborations. Annals of Tourism Research, Vol. 37, No. 4, pp. 1012-1034.

Shah, J. J., Vargas-Hernandez, N. O. E., Summers, J. D., \& Kulkarni, S., 2001. Collaborative Sketching (C-Sketch) An idea generation technique for engineering design. The Journal of Creative Behavior, Vol. 35, No. 3, pp. 168-198.

Shostack, L., 1984, Designing Services that Deliver. Harvard Business Review, Vol. 62 (January-February), pp. 133-39. 